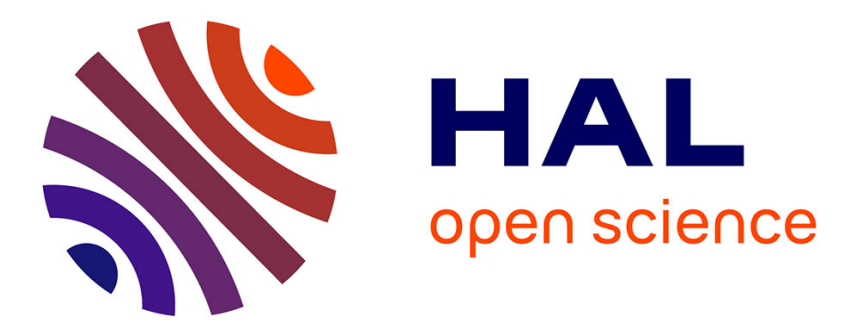

\title{
Electrostatic behaviour of space used materials in regard of internal charging met on spacecrafts
}

\author{
Thierry Paulmier, Bernard Dirassen, Romain Rey
}

\section{To cite this version:}

Thierry Paulmier, Bernard Dirassen, Romain Rey. Electrostatic behaviour of space used materials in regard of internal charging met on spacecrafts. Journal of Electrostatics, 2018, 92, pp.66-74. 10.1016/j.elstat.2018.02.002 . hal-02383078

\section{HAL Id: hal-02383078 https://hal.science/hal-02383078}

Submitted on 8 Nov 2021

HAL is a multi-disciplinary open access archive for the deposit and dissemination of scientific research documents, whether they are published or not. The documents may come from teaching and research institutions in France or abroad, or from public or private research centers.
L'archive ouverte pluridisciplinaire HAL, est destinée au dépôt et à la diffusion de documents scientifiques de niveau recherche, publiés ou non, émanant des établissements d'enseignement et de recherche français ou étrangers, des laboratoires publics ou privés. 


\title{
Electrostatic behaviour of space used materials in regard of internal charging met on spacecrafts
}

\author{
T. Paulmier*, B. Dirassen, R. Rey \\ ONERA- The French Aerospace Lab, F-31055, Toulouse, France
}

This paper is dedicated to the characterisation of charging behaviour of space used polymers in space electron environment. Spacecrafts are indeed submitted in Medium Earth Orbit (MEO) to severe fluxes of electrons with energies ranging from a few keV to several MeV. For qualification of materials used on satellites and the pre-diction of their electrostatic behaviour in space environment, it is therefore important to tests these materials in representative environment. These experiments have been carried out at ONERA, The French Aerospace Lab (Toulouse, France) in the SIRENE facility. Different polymers (PEEK, ETFE, Kapton ${ }^{\circ}$, polyurethane and silicone varnish, polyurethane based paint) have been characterised to extract the main electric parameter that steer their charging behaviour in space environment: the radiation induced conductivity (RIC). These materials have then been tested in representative environment so as to study the evolution of their conductivity and charging behaviour as a function of the received radiation dose (for low and high dose levels). From these experiments, it was possible to extract the physical parameters that steer RIC and assess numerically their charging levels in specific MEO environment.

\section{Introduction}

Materials used on spacecraft have to cope with strong level of electron and protons fluxes. These charged particles impinge on the external surfaces of the spacecraft. The high energy particles can come through the first external surfaces to get implanted within the inner dielectric parts of the spacecraft (see Fig. 1). This irradiation process induces internal electric charging on the insulating and dielectric parts of the spacecraft, with potentially high charging kinetics (if strong electron fluxes are met, like in MEO [Medium Earth Orbit] environment for instance for GPS [Global Positioning System], GLONASS [Global Navigation Satellite System] or Galileo spacecrafts) [1,2]. Different anomalies ascribed to internal charging and consequent electrostatic discharges induced by the environment have already been observed on different spacecrafts [3,4]. The electric potentials built up at the surface of the irradiated materials depend on the electric properties of these materials (bulk and surface electric conductivity, dielectric permittivity, secondary electron emission yield). Electron irradiation can therefore yield to significant potential differences between adjacent elements, which can generate high electric fields and the initiation of surface or bulk electrostatic discharge and electric arc that may induce different anomalies in the spacecraft: electromagnetic disturbances, damages on electronics and alteration of the electric properties of dielectric materials.

Space used dielectric materials present very specific behaviour in space environment due to the effect of high energy particles that come through the material and affect their electric properties due to ionisation and ageing processes. Strong differences between polymer materials like Kapton ${ }^{\circ}$ and Teflon ${ }^{\circ}$ have been observed experimentally under representative GEO (Geostationnary Earth Orbit) electron irradiation [5]. For instance, Kapton is highly conductive in this condition and becomes more and more conductive with the increasing radiation dose. On the contrary, Teflon Fluorinated Ethylene Propylene (FEP) exhibits a non-monotonous charging behaviour with a radiation induced conductivity that tends to decrease with the increasing radiation dose after a given dose threshold. We can then notice very specific behaviour under GEO like irradiation in comparison to what could be observed when materials are only irradiated with low energy electrons [5].

Most of the polymers tend to become more conductive with irradiation. The electrical conductivity is enhanced by high energy electron irradiation through ionisation processes; this is commonly referred to as radiation induced conductivity (RIC) $[6,7,8]$. The induced conductivity in polymers is well documented both experimentally and theoretically [5-13]. Space material are therefore submitted to charge implantation (due to low energy electrons) and ionisation process that tends to further charge leakage.

\footnotetext{
* Corresponding author.

E-mail address: thierry.paulmier@onera.fr (T. Paulmier).
} 


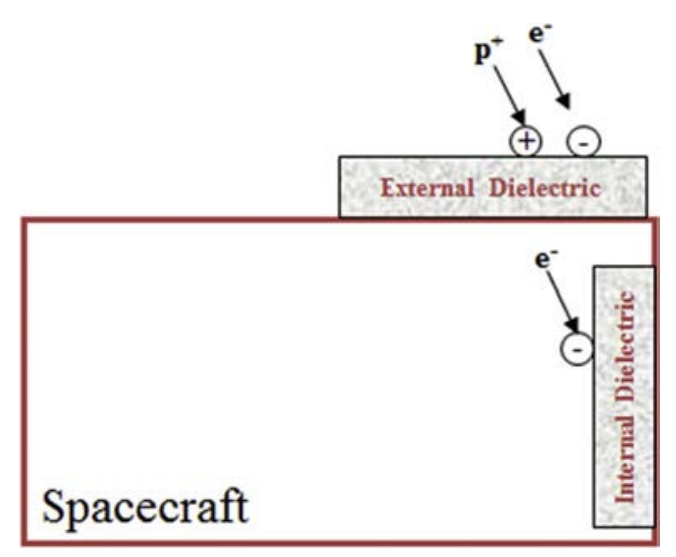

Fig. 1. Overall view of surface and internal charging process on irradiated spacecraft.

Radiation induced conductivity evolves with radiation dose rate and radiation dose injected within the material. In most conventional code, RIC is supposed to be only dependent on radiation dose rate and follows the relation below [6]:

$R I C=k_{R I C} \cdot(d D / d t)^{\Delta}$

for which $\mathrm{k}$ and $\Delta$ are empirical parameters depending on the irradiated material, $\mathrm{D}$ the injected radiation dose and $\mathrm{dD} / \mathrm{dt}$ the radiation dose rate.

RIC has been described through specific physics models used for the prediction of charging behaviour of space materials under continuous and long electron irradiation $[5,8,9]$. High energy electrons transfer a large quantity of energy within the irradiated material that allows extracting electrons from the Valence band to the conduction band (the remaining electron shortage in the Valence band is named a "hole"). Both charged particles (electrons and holes), once injected and generated in the conduction band, can get trapped in physical and chemical defects, detrapped from these defects and get recombined with an opposite charge carrier. The kinetics of trapping, detrapping and recombination of both charge carriers (electron and holes) strongly varies from one material to the other yielding to very specific behaviour for each tested material [2]. Furthermore, free charge carrier can dwell for a long period of time (up to several years) with the material. Effect of radiation dose does then prevail after the end of the irradiation process: we speak about delayed RIC (DRIC) [10,11]. DRIC can keep up high levels for Kapton ${ }^{\circ}$ for a long period of time while it fades away quite quickly for Teflon ${ }^{\circ}$ FEP. Successive irradiations can then present different profiles depending on DRIC relaxation kinetics: for Teflon ${ }^{\circledR}$ FEP, for instance, DRIC fades away quite quickly. But this quick decline does not induce total recovery: electron trapped during previous irradiations act as recombination centers for the following irradiations, leading to a decrease of the effective generation rate and therefore to a decline of RIC [12]. For Kapton however, decline of DRIC is very slow and Kapton keeps up therefore high conductivity for a long period of time without any reversed RIC since trapped charge carrier can get detrapped easily: this material tends therefore to get more and more conductive [13].

The charging behaviour of space used materials can therefore be very different from one material to the other. It is thus important to take great care on the characterisation process of the different involved materials for a good and realistic prediction of their charging level in space environment.

The objective of the current study performed at ONERA (Toulouse, France) is then to assess the conductivity level of space used insulating materials in representative electron flux and calculate, through a dedicated model, the charging of materials in representative electron environment. The characterisation step includes extraction of intrinsic bulk conductivity and radiation induced conductivity (RIC). The effect of delayed RIC (DRIC) on the long term charging behaviour was also

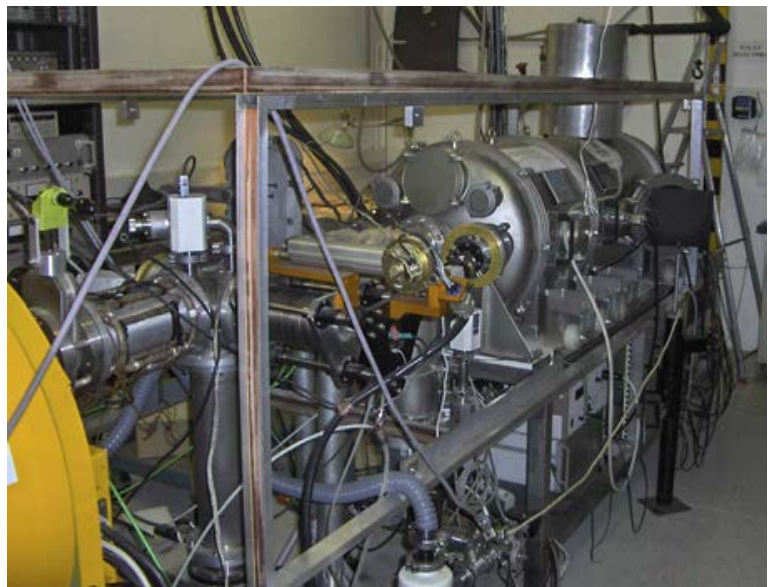

Fig. 2. View of the SIRENE irradiation facility.

analysed on different space used materials. The influence of these different conductivities has been assessed on all defined materials so as to be able to predict charging behaviour in very different irradiation conditions. The physical model used for charging prediction will be presented followed by a validation and the use of this model for charging prediction of PEEK in averaged MEO conditions.

\section{Experimental set-up and protocol}

\subsection{Irradiation facility}

SIRENE (Fig. 2) is a sophisticated and unique test facility especially designed for the study of surface and internal charging of space materials and satellite components under extreme environment (usually geostationary orbit radiation conditions). This facility is able to reproduce the geostationary electron spectrum (in the energy range [0-400 keV]). It allows realistic assessment of potential built up in geostationary orbit. Its flexibility allows the simulation of extreme environments different than the geostationary one (eg, MEO, LEO [Low Earth Orbit] or other planetary radiation environments). This facility is widely used as well for the characterisation of radiation induced conductivities of space materials in vacuum. Using the high energy electron beam and a high incoming flux on the sample (up to $10 \mathrm{nA} \mathrm{cm}^{-2}$ ), we are able as well to perform radiative ageing on the material. Space materials, such as polymers, adhesive or coverglasses can then be aged in SIRENE with equivalent flight durations equal to several months or years. The electron spectrum simulation is achieved by the use of two monoenergetic electron beams ( 20 and $400 \mathrm{keV}$ ), these two beams being diffused in energy and angle to produce a space-like electron flow with a good flux homogeneity in a diameter equal to $20 \mathrm{~cm}$ on the sample holder. The nominal integrated fluxes used for the $20 \mathrm{keV}$ monoenergetic beam and the distributed $0-400 \mathrm{keV}$ one are respectively equal $250 \mathrm{pA} \mathrm{cm}^{-2}$ and $50 \mathrm{pA} \mathrm{cm}^{-2}$ but these different fluxes can be changed independently. The temperature of the sample holder can be controlled in the range $\left[-180^{\circ} \mathrm{C},+250{ }^{\circ} \mathrm{C}\right]$ allowing to reproduce the temperature variations of materials on flight. A pumping system allows experiments at vacuum of around $10^{-6} \mathrm{hPa}$. It is instrumented with a contact-less electrostatic probe, current measurement systems and noncontact PEA [Pulsed Electro-Acoustic] device. Thanks to these different experimental specifications (bulk conductivity and RIC characterisation, temperature effect) and its spectrum flexibility, the SIRENE experimental facility is highly relevant for the current study, especially regarding the characterisation of bulk conductivity and RIC as well as delayed ionisation effects (DRIC) dose on the different selected materials. SIRENE can be connected as well to a Transfer Unit which enable the transfer of samples irradiated in SIRENE to a vacuum storage unit or to other irradiation facilities. This sample transfer is performed under 
vacuum without any vacuum break-up that would induce possible recovery or discharging effects.

\subsection{Tested samples}

The material tested in this current study have been selected according to their potential risk in term of charging. The most critical parts within the satellite for which the occurrence of an electrostatic discharge would lead to potentially hazardous disturbances or damages are elements used for electric and electronic applications or for large surface area that would strongly steer the overall internal charging potential of the satellite. Materials selected for the experimental tests have therefore been classified accordingly.

- Polyetherimide (PEI): high performance polymer used for mechanical support and for electrical insulation. PEI samples tested here are $100 \mu \mathrm{m}$ PEI sheets.

- Polyetheretherketone (PEEK): high performance polymer used for mechanical support, thermal and electrical insulation. PEEK samples tested here are $100 \mu \mathrm{m}$ PEEK sheets

- Ethylene tetrafluoroethylene (ETFE): polymer used especially for electrical insulation for cable and wire. ETFE samples tested here are ETFE wires.

- Polyimide Kapton ${ }^{\circledR}$ : high performance polymer used for thermal and electrical insulation. Kapton samples tested here are Kapton wires.

- Polyurethane (Solithane ${ }^{\circledast}$ ) and silicone (MAPSIL 213 ${ }^{\circledast}$ ) films: these materials are used as potting, varnish and conformal coating on electronic components and boards. The samples tested here are $100 \mu \mathrm{m}$ thick films deposited on aluminium substrates.

- Polyrethane PU1 black paint: used as paint for thermal control. The samples tested here are $100 \mu \mathrm{m}$ thick films deposited on aluminium substrates.

\subsection{Experimental protocols}

The overall objective is to understand the physical mechanisms that rule the charging behaviour of materials in space environment. For this purpose, the first task is to extract intrinsic and radiation induced conductivity for different radiation dose rates for these materials. The second task is to study the influence of radiation dose on instantaneous RIC and analyse delayed effect of radiation dose so as to be able to take into account radiation history for the evaluation of charging risks on components. The basic approach for this second task is to put into evidence delayed RIC. Ageing effect (for high radiation dose levels) on the evolution of RIC was also taken into account. The tests have been performed in the SIRENE facility installed at ONERA. Both tasks are described thoroughly in the following sub-sections.

\subsection{1. - Analysis of conduction processes (extraction of bulk conductivity} and RIC)

Extraction of bulk conductivity and RIC on the different tested materials has been performed following the conventional irradiation method applied at ONERA [13]. For the evaluation of the intrinsic bulk conductivity in this project, we proposed then to apply the potential decay method. The experimental procedure is summarised hereafter:

- The sample is first exposed to charging by the means of the low energy electron beam ( $20 \mathrm{keV}$, Beam 1 of Fig. 3).

- The sample irradiation is then shut down, and its surface potential continuously monitored by use of an electrostatic (contact-less) surface voltmeter (TREK 341B) connected to a non-contact Kelvin probe (TREK 3455ET), yielding to the decrease of surface potential versus relaxation time, $\mathrm{Vs}(\mathrm{t})$. The non-contact Kelvin probe is place at $5 \mathrm{~mm}$ in front of the samples and move along the different samples with a motorised translation system.

- The model of a capacitor relaxing its charge through a resistance

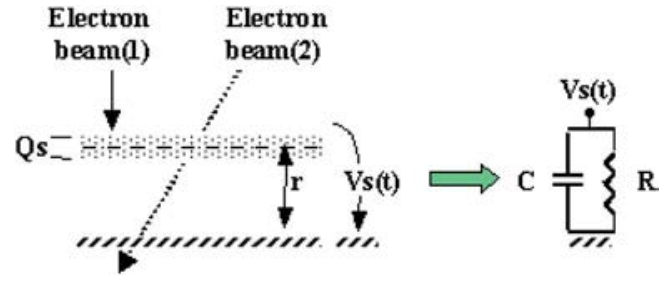

Fig. 3. The surface voltage decay method for intrinsic and radiation induced conductivity evaluation (electron beam 1: charging beam - electron beam 2: decay stimulating beam for RIC assessment).

(see Fig. 3) is then applied. The time constant for charge leakage (RC) was determined and ultimately the resistivity $\rho(\Omega . m)$ or the inverse, its conductivity $\sigma$ [13].

$\sigma=\varepsilon_{0} \varepsilon \cdot \frac{d v_{s} / d t}{V_{s}}(\Omega \cdot \mathrm{mk})^{-1}$

for which $\mathrm{V}_{s}$ is the surface potential measured on the sample, $\varepsilon$ the dielectric constant of the material, $\varepsilon_{0}$ the vacuum permittivity and $t$ is the time.

In the conductivity evaluation approach, when the surface potential is allowed to decay "naturally" (Beam 2 on Fig. 3 is not used), the end result is the so called intrinsic or dark conductivity. For space application, the charge decay method is usually applied for RIC measurements. However, instead of letting the charges leak "naturally" in the bulk, a penetrating ionizing beam (Beam 2 on Fig. 3) is now used to stimulate the decay. This electron beam is used to enhance the conductivity (initiating then RIC) without inducing any charge implantation. In this task, $100 \mu \mathrm{m}$ thick samples have been tested and an energy of $400 \mathrm{keV}$ for the high energy part has been applied in the SIRENE facility. We are then able to reach a "complex" conductivity, which according to earlier works is expressed as the sum of two terms, dark conductivity, and radiation induced conductivity [13].

$\sigma_{t}=\sigma_{\text {Dark }}+\sigma_{R I C}$, and $\sigma_{r i c}=k\left[\frac{d D}{d t}\right]^{\Delta}$

where $\sigma_{\text {dark }}$ is the intrinsic bulk conductivity and $\sigma_{\text {RIC }}$ the radiation induced conductivity. $\mathrm{dD} / \mathrm{dt}$ is the radiation dose rate (in Gy.s ${ }^{-1}$ ) while $\mathrm{k}$ and $\Delta$ are empirical parameters depending on the tested material [13].

Most of the time, RIC dominates over DARK for materials with very low initial levels of (dark) conductivity. To determine the $\mathrm{k}$ and $\Delta$ factors, these measurements must be repeated at three different dose rates. The $\mathrm{k}$ and $\Delta$ parameters can be extracted by representing Ln $\left(\sigma_{\text {RIC }}\right)$ as a function of $\mathrm{Ln}(\mathrm{dD} / \mathrm{dt})$. Using equation (3), we can then write $L n \sigma=L n k+\Delta L n(d D / d t)$ and then derive numerically $\mathrm{k}$ and $\Delta$. RIC and/or bulk conductivity have been extracted on the seven different materials described above.

\subsection{2. - Cumulative effect of radiation dose on RIC and DRIC}

Charging behaviour of space used materials is very complex and equilibrium is usually not reached on the induced surface potential [15]. This is due to the fact that RIC evolves with radiation dose and its behaviour strongly differs from one material to the other. Moreover radiation dose effect may prevail for a long period of time after irradiation shut-down which yields to the fact that no recovery of the initial charging behaviour is observed during successive irradiations for several space used materials (especially polymers). For satisfactory charging prediction, it is of high importance to characterise this cumulative dose effect and delayed effect on RIC for the different defined materials. The experimental approach is the measurement of surface potential profile (as a function of irradiation time and radiation dose) on the different defined materials in the SIRENE facility under double electron beam irradiation: $20 \mathrm{keV}, 100 \mathrm{pA} \mathrm{cm}^{-2}+400 \mathrm{keV}, 50 \mathrm{pA} \mathrm{cm}^{-2}$. The first electron beam induces charge implantation within the sample 
while the high energy electron beam induces ionisation of the overall bulk (and therefore dose injection) without charge implantation. Three consecutive irradiation steps have been performed, separated by $24 \mathrm{~h}$ relaxation. This experimental approach enables to analyse the possible dependence of RIC of each material with radiation and any ageing effect under high radiation dose. For this last point, following the three successive above irradiation, the same samples have been irradiated under $400 \mathrm{keV}$ electrons at $5 \mathrm{nA} \mathrm{cm}{ }^{-2}$ for $5 \mathrm{~h}$, which corresponds to an averaged injected dose in the bulk equal to around $3.10^{5} \mathrm{~Gy}$. For internal charging issue, this dose level is equivalent to the dose received by the materials behind $1 \mathrm{~mm}$ shielding with the AE8 MEO electron spectrum [14] close to the surface (at $2 \mu \mathrm{m}$ depth) after 10 years on flight. The aged samples have then been submitted to the same double beam irradiation $\left(20 \mathrm{keV}, 100 \mathrm{pA} \mathrm{cm}^{-2}+400 \mathrm{keV}, 50 \mathrm{pA} \mathrm{cm}^{-2}\right)$ so as to analyse any possible evolution of their electric conductivity.

Three different materials have been tested in this step: PEI, PEEK and Kapton wires.

\section{Experimental results}

\subsection{Analysis of conduction processes}

Fig. 4 presents the evolution of the surface potential on different materials along the different steps of the test: charging, intrinsic relaxation and stimulated relaxation with the $400 \mathrm{keV}$ electron beam. We can notice on this figure that all materials gets charged under $20 \mathrm{keV}$ electron beam except for the Polyurethane PU paint that do not present any charging level at all, meaning that its bulk intrinsic conductivity is higher than $10^{-12} \Omega^{-1} \mathrm{~m}^{-1}$. On the contrary, intrinsic relaxation is very low for several materials (PEI, PEEK, ETFE, Kapton ${ }^{\circ}$ ) meaning that their intrinsic bulk conductivity is very low, lower than $10^{-15} \Omega^{-1} \mathrm{~m}^{-1}$, which explains the reason why these materials gets significantly charged at $20 \mathrm{keV}$. Solithane intrinsic relaxation kinetics is slightly higher, yielding to a bulk conductivity equal to $10^{-14} \Omega^{-1} \mathrm{~m}^{-1}$. The silicon varnish MAP 213 presents a fairly high relaxation kinetics linked to a higher conductivity equal to $3.10^{-14} \Omega^{-1} \mathrm{~m}^{-1}$.

However, the most important parameter is radiation induced conductivity (RIC) that steers at great level the charging behaviour of materials in space environment. RIC is characterised during the last phase of this test. Solithane does not present any increase of its relaxation kinetics when the $400 \mathrm{keV}$ electron beam is turned on, and therefore possess a very low RIC (in comparison with bulk conductivity). For ETFE, for which bulk conductivity is low, the situation is more complex: as time increases, we can observe a steady rise of the relaxation kinetics. RIC seems to be very low at the start but seems to be increasing with the increasing radiation dose. However, this RIC keeps up very low levels for a long period of time meaning that this material is prone to present significant charging levels behind shielding. The other materials (PEI, PEEK, MAP 213, Kapton) present a significant rise of the relaxation kinetics when the $400 \mathrm{keV}$ irradiation process is initiated: this rise reveals a noticeable RIC for the different materials. However, their behaviour in space environment might be more complex than for ETFE or PU paint, due to the influence of dose on RIC, as we will see in the next section.

Fig. 5 presents the evolution of RIC as a function of time during the stimulated relaxation process. As time increases, the electric field drops down but radiation dose increases. This figure is therefore a picture on the dependence of RIC with electric field and radiation dose. As observed earlier, ETFE presents a constant increase of RIC (after $10 \mathrm{~min}$ irradiation) with radiation dose during the first $30 \mathrm{~min}$ relaxation and reaches a constant level after this duration. During the first $10 \mathrm{~min}$, RIC of ETFE slightly drops down revealing as well a dependence of RIC with the electric field (which decreases during relaxation). After $10 \mathrm{~min}$, the effect of dose prevails over the effect of electric field yielding to a change of RIC behaviour. For the other materials that presents a noticeable RIC (PEI, Kapton ${ }^{\circ}$, PEEK), we can observe a steady decline of RIC with irradiation time that should be ascribed, as demonstrated previously at ONERA [2], by a dependence of RIC with electric field. These results show the importance to take into account effect of radiation dose and electric field on charge transport and ionisation processes (in physical and numerical models) for a correct prediction of charging kinetics in space environment. For MAP 213, it was difficult to analyse evolution of RIC along the irradiation process, due to the steep relaxation kinetics that yield to strong errors after few minutes irradiation.

The $\mathrm{k}$ and $\Delta$ parameters linked to RIC have been extracted for PEI, PEEK, MAP 213 and Kapton wires applying four different successive $400 \mathrm{keV}$ electron radiation dose rates at $0.24,2.4,24$ and $121 \mathrm{mGy} \mathrm{s}^{-1}$ (for silicon).

We have noticed that PEEK presents a $\Delta$ parameter equal to 1 , meaning that increasing radiation dose rate (or electron flux) by a factor $\alpha$ would lead to an enhancement of RIC by the same factor. The consequence on charging kinetics is interesting: PEEK will present the same charging potential between one given electron spectrum and another one (shifted in flux by a factor $\alpha$ in comparison to the first spectrum): indeed, we would increase charging current and conduction current by the same factor. Spectrum effect on PEEK will be lower than for the other materials for which the $\Delta$ parameter is lower than 1 .

It was interesting as well to notice that the $\Delta$ parameter for Kapton wire is much lower than $1(0.68)$ contrary to what was extracted for Kapton $^{\circ} \mathrm{HN}$ ( $\Delta$ parameter close to 1 ) [4]. This can be due to the material processing or slight differences on the structure of both materials.

\subsection{Analysis of cumulative effect of radiation dose}

The second step of phase 1 was to study the instantaneous and delayed effect of cumulative radiation dose on conductivity of different

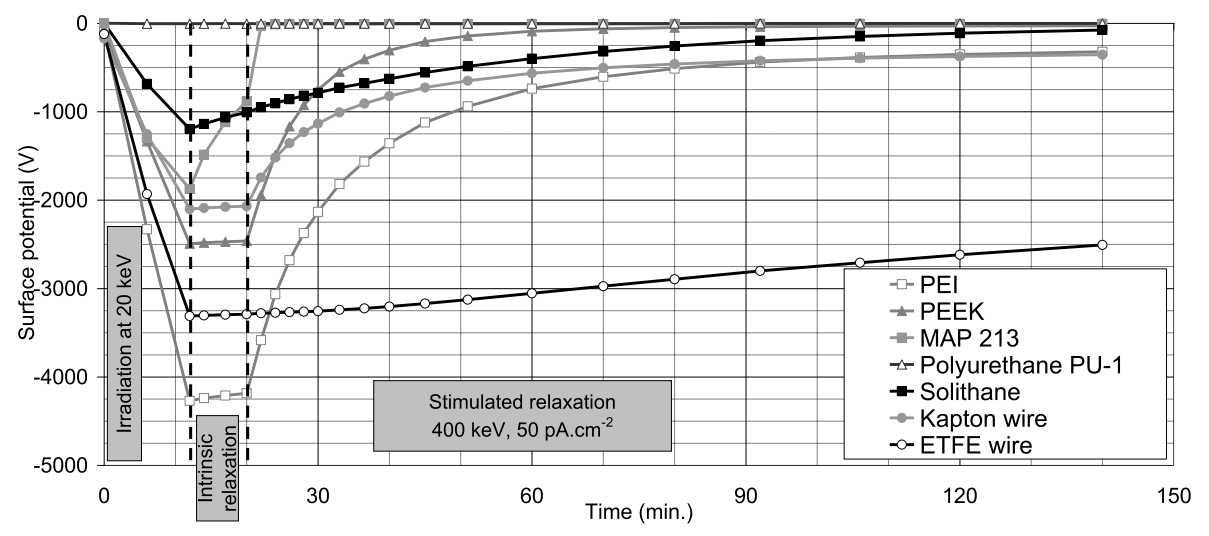

Fig. 4. Evolution of surface potential on the different material sample as a function of time along the three different phases. 


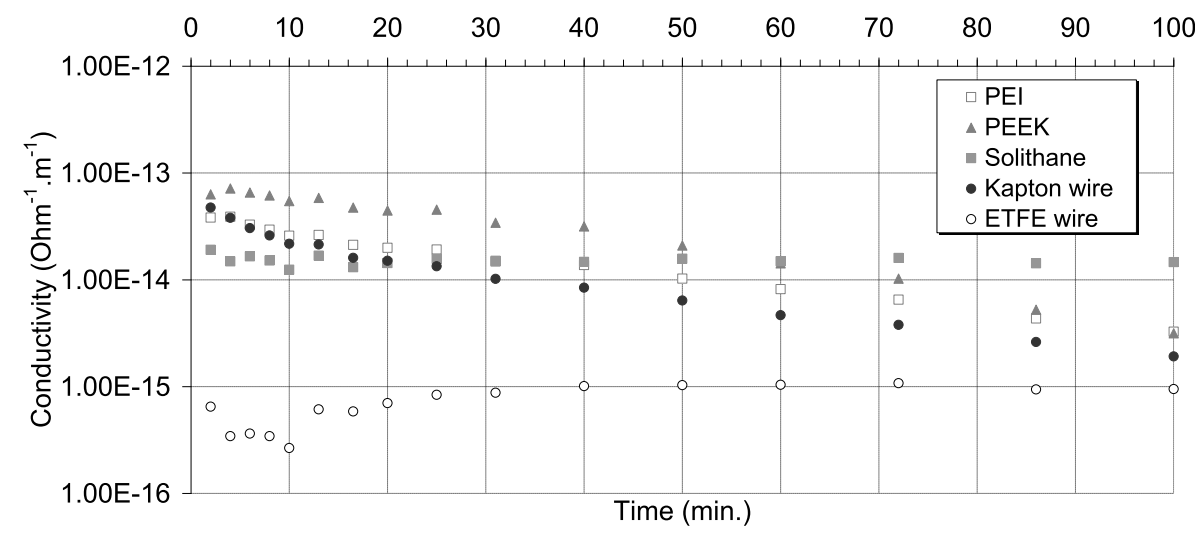

Fig. 5. Evolution of radiation induced conductivity as a function of time during the stimulated relaxation phase at $400 \mathrm{keV}$.

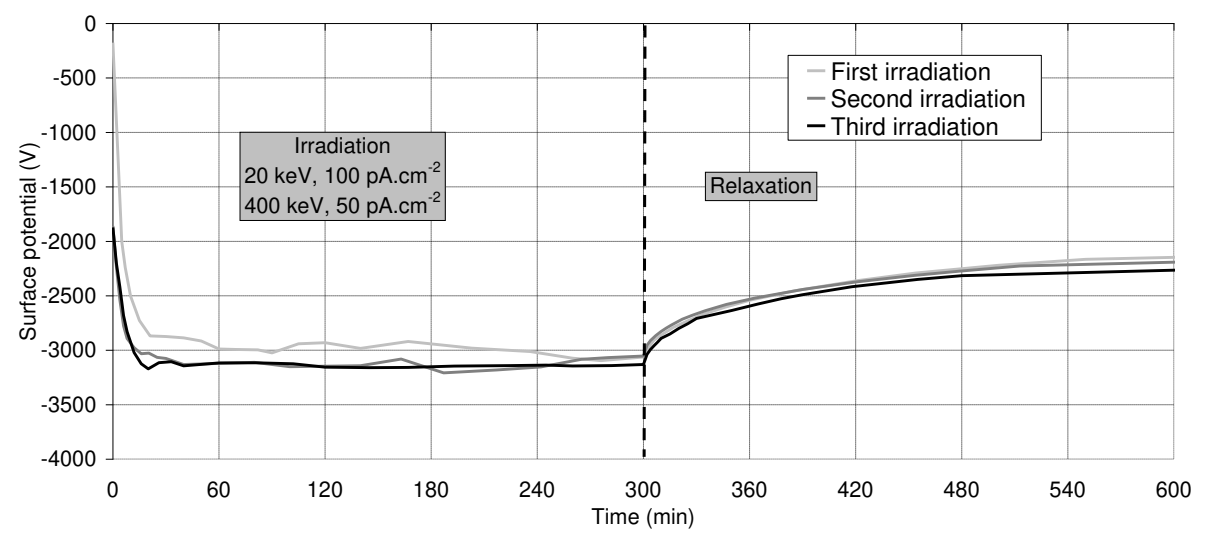

Fig. 6. Evolution of surface potential as a function of irradiation time on PEI during the three successive irradiation steps.

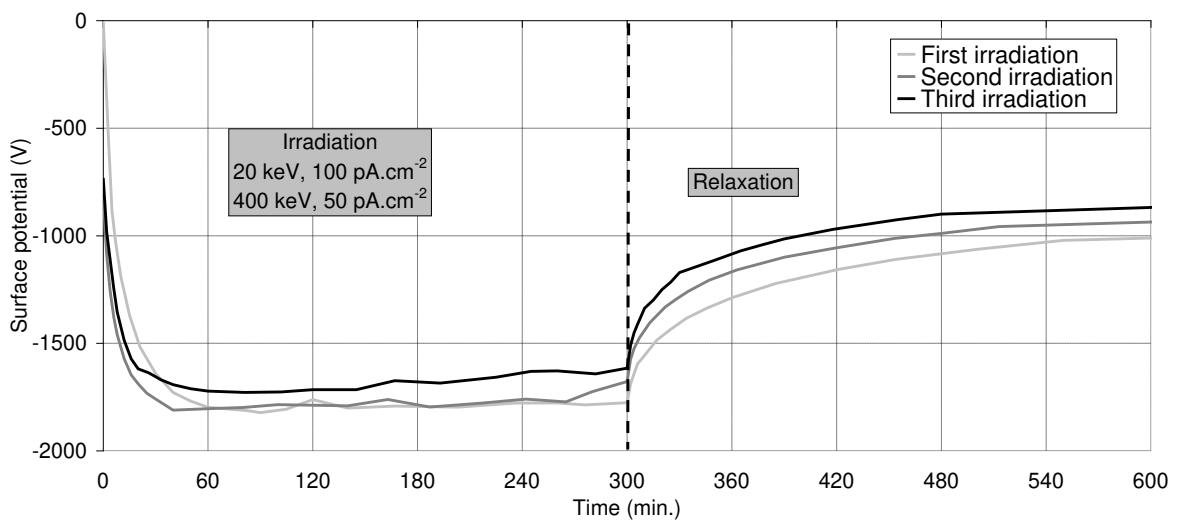

Fig. 7. Evolution of surface potential as a function of irradiation time on Kapton wires during the three successive irradiation steps.

materials presenting significant RIC: PEI, PEEK, Kapton wires. For this purpose, these materials have been submitted to three successive irradiation steps with an electron spectrum $(20 \mathrm{keV}+400 \mathrm{keV})$ producing charging and ionisation. Figs. 6-8 present the evolution of surface potential during irradiation and relaxation for the three successive steps on the different materials (19h relaxation between each irradiation steps). The surface potential on PEI and PEEK is not strongly affected during the three successive irradiation steps: we can only observe a slight shift to higher absolute surface potential, corresponding to a small degradation of the effective electric conductivity. This slight shift is explained as well by the fact that radiation induced conductivity quickly fades away after irradiation: the relaxation kinetics of the surface potential on PEEK is then very low and this surface potential did not reach zero before the start of the second irradiation. This steep decline of RIC during relaxation is due to the implantation of trapped charges (produced by ionisation) that act as recombination centers for the electron-hole pairs (generated as well by ionisation) which reduces then the density of free charges in the material during relaxation and for the next irradiation step [xv]. Kapton wires tends to get slightly more conductive with the successive irradiation steps (see Fig. 7): with the increasing radiation dose, density of free charges for Kapton rises steadily leading to a constant rise of RIC with dose. Looking at one irradiation step, we can indeed observe a constant decline of the absolute surface potential along the irradiation. This process has already been observed on Kapton ${ }^{\circ}$ HN films, as described in Ref. [13].

The next step was to analyse any possible ageing effect when materials have received a high radiation dose level. PEI, PEEK and Kapton wires have then been submitted to a total radiation dose equal to $3.10^{5}$ Gy and irradiated again with an electron spectrum $(20 \mathrm{keV}+400 \mathrm{keV})$, as for pristine materials. Figs. 9-11 presents the 


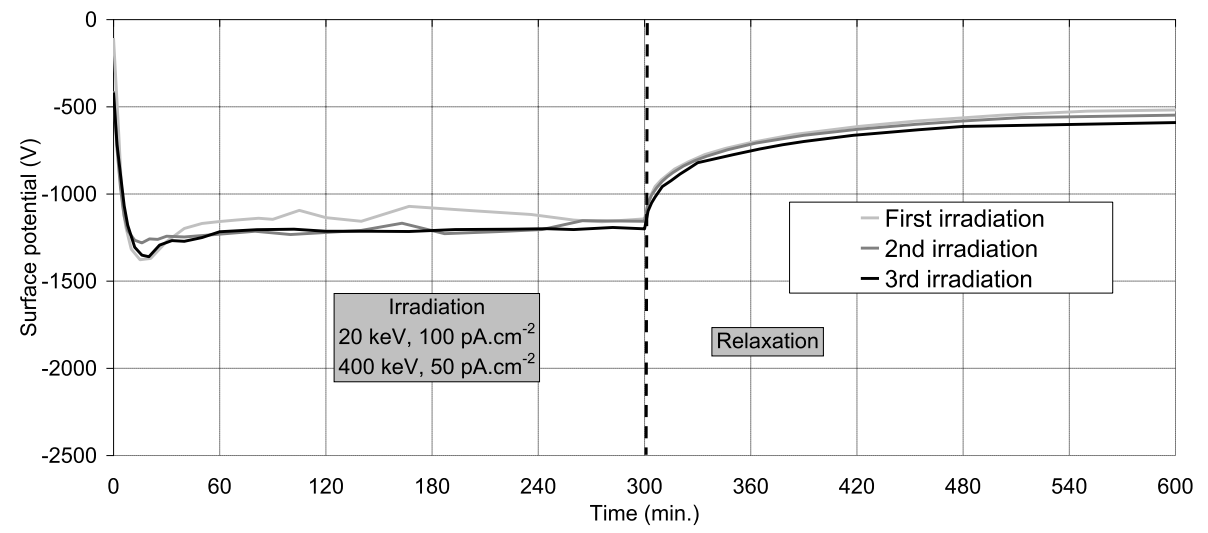

Fig. 8. Evolution of surface potential as a function of irradiation time on PEEK during the three successive irradiation steps.

evolution of surface potential on pristine and aged samples for the three materials. We can clearly observe a significant shift of the surface potential for PEI and PEEK to higher levels which is linked to a decline of RIC for these two materials. This trend has been observed on several space used polymers (silicon, PEEK, FEP, ETFE, ...) and is usually due to crosslinking effects together with the creation of new chemical bonds that reduce molecular mobility and charge transport. This conductivity decrease has been demonstrated through different in-situ and ex-situ measurements and a former study performed on space used silicons proved also a steep decline of conductivity $[16,17]$. On the contrary, Kapton wires tends to get more conductive with the increasing radiation dose (for doses lower than $10^{6} \mathrm{~Gy}$ ): this result confirms previous studies on Kapton ${ }^{\circ} \mathrm{HN}$ on which we observed the same trend. This feature was ascribed to the high generation of free charges that prevail for a long period of time in the extended states and to the high resistance of Kapton to radiation doses [18].

Space materials might therefore behave very differently over a long period of time under radiation. Despite the presence of RIC that can reduce charging kinetics, it is important as well to take into account the effect of radiation dose that may affect conductivity and increase risk of charging. In this context, it is important to perform numerical prediction that are based on physical models taking into account not only RIC but also the effect of radiation dose and electric field on ionisation and charge transport. A physical model has been developed at ONERA to comply with these requirements. This is the topic of next section.

\subsection{Numerical analysis}

A dedicated physical and numerical model, called RC (Resistance/ Capacitance) model, has been developed to describe the evolution of surface potential and RIC of space-used materials irradiated with highenergy electrons in representative charging conditions. This model is based on former studies and models devised by Rose [19] and Fowler [6]. In this physical model, the irradiated sample is modelled as a system of a capacitance and an electrical resistance in parallel. From this simple model, the surface potential can be calculated by the relation:

$\frac{d V}{d t}=\frac{L j(1-\eta-\beta)-e\left(\mu_{n} n+\mu_{p} p\right) V}{\varepsilon} \overrightarrow{A B}$

in which $\mathrm{L}$ is the sample thickness, $\mathrm{J}$ the incident flux of low energy implanted electrons ( $20 \mathrm{keV}), \eta$ the secondary electron emission yield, $\beta$ the surface leakage parameter, $\mathrm{V}$ the surface potential, e the elementary charge, $\varepsilon$ the material permittivity, $n$ density of free electrons and $p$ densities of free holes (vacancies of electrons).

The density of free charges can be assessed with the equations of the circuit RIC model:

$$
\begin{aligned}
& \frac{d n}{d t}=g(E, T)-\alpha_{1} n\left(p_{t 1}+p_{t 2}\right)-\frac{n}{\tau_{n 1}}-\frac{n}{\tau_{n 2}}+\frac{n_{t}}{\tau_{n t 1}}+\frac{n_{t 2}}{\tau_{n t 2}} \\
& \frac{d p}{d t}=g(E, T)-\alpha_{2}\left(n_{t 1}+n_{t 2}\right) p-\frac{p}{\tau_{p 1}}-\frac{p}{\tau_{p 2}}+\frac{p_{t}}{\tau_{p t 1}}+\frac{p_{t 2}}{\tau_{p t 2}}
\end{aligned}
$$

$\frac{d n_{t 1}}{d t}=\frac{n}{\tau_{n_{1}}}-\frac{n_{t 1}}{\tau_{n t_{1}}}-\alpha_{2} p n_{t 1}$

$\frac{d n_{t 2}}{d t}=\frac{n}{\tau_{n 2}}-\frac{n_{t 2}}{\tau_{n t 2}}-\alpha_{2} p n_{t 2}$

$\frac{d p_{t 1}}{d t}=\frac{p}{\tau_{p 1}}-\frac{p_{t 1}}{\tau_{p t}}-\alpha_{1} n p_{t 1}$

$\frac{d p_{t 2}}{d t}=\frac{p}{\tau_{p 2}}-\frac{p_{t 2}}{\tau_{p t 2}}-\alpha_{1} n p_{t 2}$

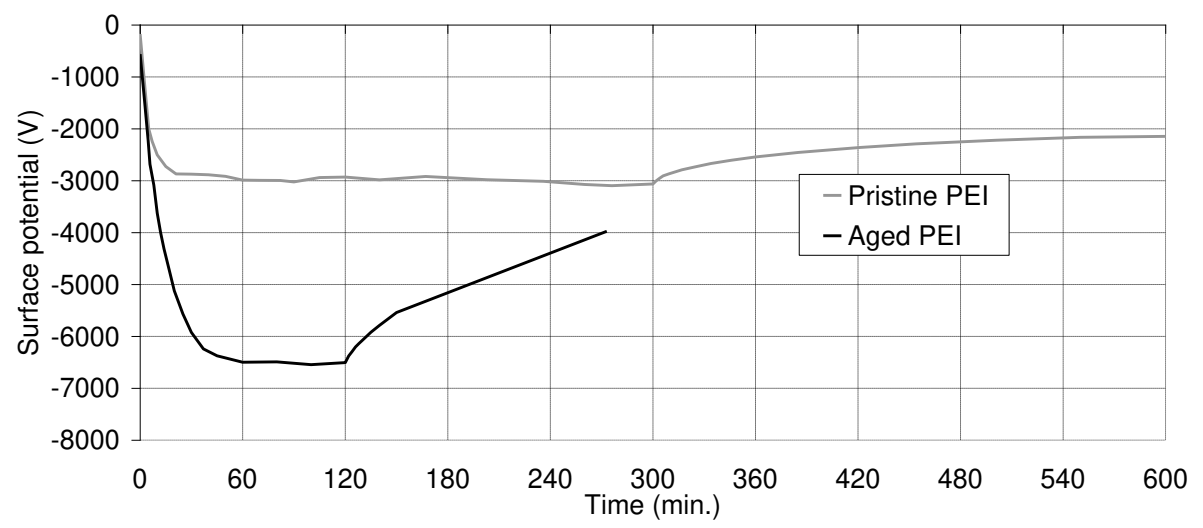

Fig. 9. Evolution of surface potential on pristine and aged PEI sample (dose for ageing: $3.10^{5} \mathrm{~Gy}$ ). 


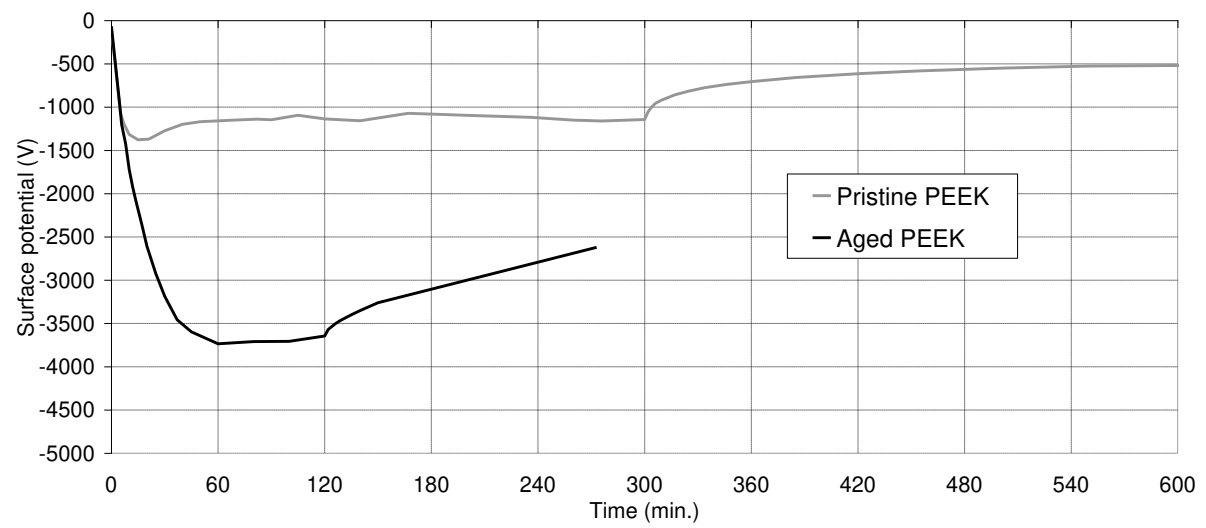

Fig. 10. Evolution of surface potential on pristine and aged PEEK sample (dose for ageing: $3.10^{5} \mathrm{~Gy}$ ).

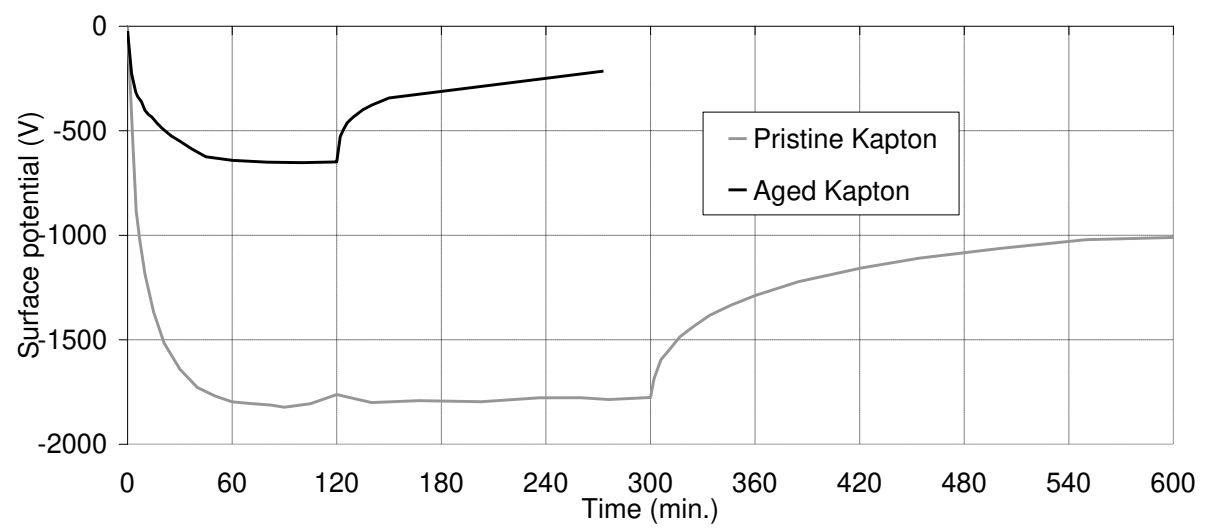

Fig. 11. Evolution of surface potential on pristine and aged Kapton wires (dose for ageing: $3.10^{5} \mathrm{~Gy}$ ).

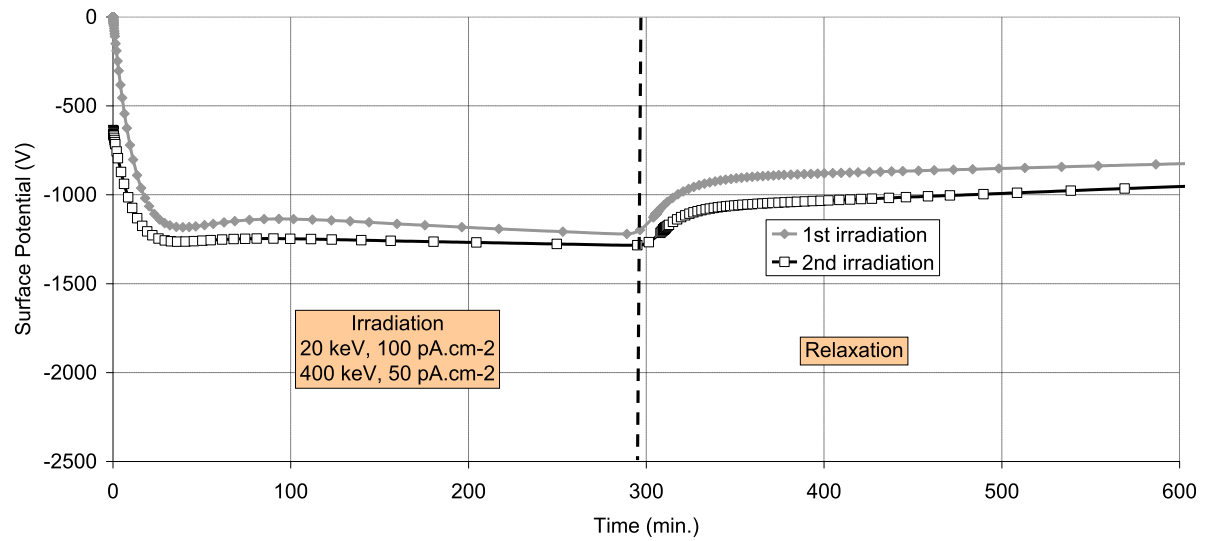

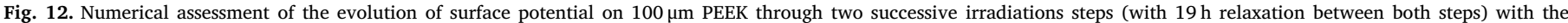
following irradiation configuration: $20 \mathrm{keV}, 100 \mathrm{pA} \mathrm{cm}^{-2}+400 \mathrm{keV}, 50 \mathrm{pA} \mathrm{cm}^{-2}$.

for which $g(E, T)$ is the electron/hole pairs generation rate dependent on electric field and temperature, $\alpha_{1}$ and $\alpha_{2}$ are the recombination rates, $\mathrm{n}, \mathrm{n}_{\mathrm{t} 1}$ and $\mathrm{n}_{\mathrm{t} 2}$ are densities of free and trapped electrons, $\mathrm{p}, \mathrm{p}_{\mathrm{t} 1}$ et $\mathrm{p}_{\mathrm{t} 2}$ are densities of free and trapped holes, $\tau_{\mathrm{n} 1}, \tau_{\mathrm{n} 2}, \tau_{\mathrm{p} 1}$ and $\tau_{\mathrm{p} 2}$ are trapping time for free electrons and holes, $\tau_{\mathrm{nt} 1}, \tau_{\mathrm{nt} 2}, \tau_{\mathrm{pt} 1}$ and $\tau_{\mathrm{pt} 2}$ detrapping time for trapped electrons and holes.

From these different equations, we can then assess the evolution of surface potential of the irradiated materials as a function of irradiation and relaxation time. This approach has been applied on PEEK.

The first step was to extract from the previous experimental tests the different parameters needed to assess the density of free charges: trapping times, detrapping times, recombination coefficients, generation rate. This extraction phase has been performed with a numerical code developed at ONERA which is based on an error optimization algorithm with gradient method.

In a second step, these parameters have been implemented in the RC model for the evaluation of surface potential of a $100 \mu \mathrm{m}$ thick PEEK sample irradiated in the same conditions as those applied experimentally: double electron beam $\left(20 \mathrm{keV}, 100 \mathrm{pA} \mathrm{cm}^{-2}+400 \mathrm{keV}\right.$, $50 \mathrm{pA} \mathrm{cm}^{-2}$ ) with two successive irradiations $(19 \mathrm{~h}$ relaxation between each irradiation step). Fig. 12 presents the numerical results with the above irradiation configuration. We can notice that the model results fits quite well with the experimental values (in comparison with Fig. 8). During the second irradiation step, we can notice a slight shift of the surface potential to lower values, which is due, as stated previously, to an initial potential that is not zero (but equal to $-640 \mathrm{~V}$ ) at the start of 


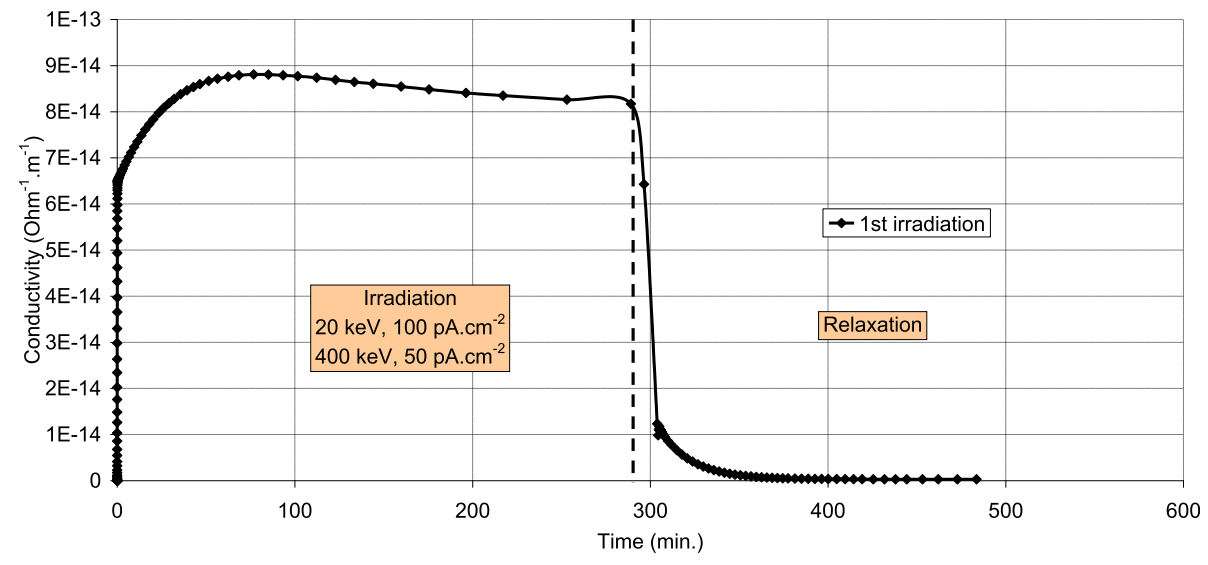

Fig. 13. Numerical assessment of the evolution of radiation induced conductivity on $100 \mu \mathrm{m}$ PEEK during the first irradiation and relaxation process.

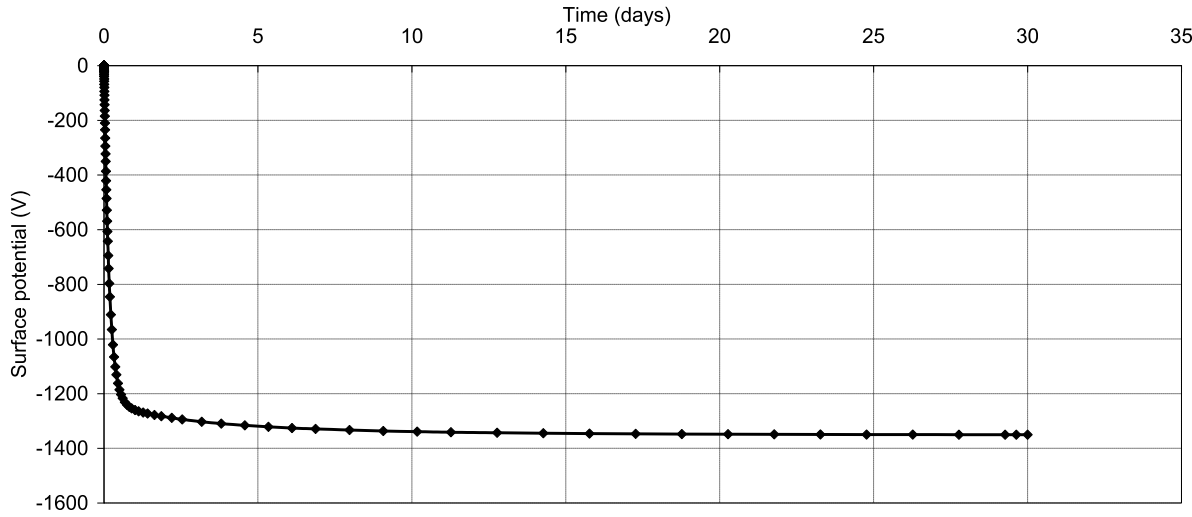

Fig. 14. Numerical assesment of the evolution of surface potential on $100 \mu \mathrm{m}$ PEEK under MEO electron environment (AE8) for 30 days.

the second irradiation. Both profiles present the same temporal behaviour as the experimental ones.

Fig. 13 presents the evolution of radiation induced conductivity assessed with the physical model on PEEK during irradiation and relaxation for the first irradiation step. We can notice a slight initial rise of RIC, which explains the gradual decrease of the absolute surface potential between $35 \mathrm{~min}$ and $120 \mathrm{~min}$ irradiation. After irradiation shut-down, RIC prevails for a long period of time: we speak about delayed RIC (DRIC). DRIC however steeply drops down during the first seconds of irradiation and then slowly fades away over several hours relaxation. The initial drop of DRIC is due to fast trapping of free charges generated during irradiation. The slow decline of DRIC during the second relaxation step has been ascribed to the effect of recombination between charges of opposite signs (electrons and holes) that gradually reduces the density of trapped charges and therefore the detrapping kinetics that steers, during relaxation, the density of free charges.

This physical model allows a further step by assessing the charging levels of materials in representative space environment. From the previous analysis on PEEK, it is then possible to calculate the evolution of surface potential on a $100 \mu \mathrm{m}$ thick PEEK irradiated under an averaged AE8 MEO electron environment, as seen in Fig. 14. We can then notice that this material might present fairly significant charging levels in MEO environment, even under averaged electron spectra.

\section{Conclusion}

The overall results revealed that space used materials can be submitted to strong charging levels that may affect the operation of the spacecraft especially for inner elements. Different polymer materials have been tested and numerically analysed using the physical parameters that have been extracted experimentally. The numerical model allows demonstrating strong discrepancies from one material to the other in regard of charging behaviour which is due to their different response to ionisation processes and charge transport. PEEK and Kapton wires present for instance a significant conductivity with high resistance to radiation. Others like ETFE wires do not present any significant conductivity leading quickly to steep charging kinetics under irradiation. Dedicated experiments have been defined and can be used as standard tests to extract the parameters used in different numerical models for charging predictions, taking into account radiation dose effects, charge implantation, electric field effects, ...These parameters are important as well, following the representative irradiation tests, to perform extrapolation on charging levels induced over a long period of time. A physical model has been developed to assess the charging kinetics of dielectric materials in space environment: this model yields to numerical results that fit well with the experimental data.

\section{Acknowledgments}

The research leading to these results has received funding from the European Union Seventh Framework Programme (FP7/2007-2013) under grant agreement 606716 (SPACESTORM). The authors would also like to thank CNES, the French Space Agency, for technical and scientific support over the successive R\&T studies that helped in developing specific irradiation and scientific tools for this current study.

\section{Appendix A. Supplementary data}

Supplementary data related to this article can be found at http://dx. doi.org/10.1016/j.elstat.2018.02.002. 


\section{References}

[1] A. Sicard-Piet, S. Bourdarie, D. Boscher, R. Friedel, T. Cayton, Solar Cycle Electron Environment at GNSS like Altitudes, 57th International Astronautical Congress Valencia, Spain, (2006) IAC-06-D5.2.04.

[2] J.-C. Matéo-Vélez, N. Ganuschkina, N. Meredith, A. Sicard-Piet, V. Maget, D. Payan, I. Sillanpaa, S. Dubyagin, From GEO/LEO Environment Data to the Numerical Estimation of Spacecraft Surface Charging at MEO", 14th Spacecraft Charging Technology Conference, ESA/ESTEC, Noordwijk, NL, APRIL 2016, pp. 04-08.

[3] G.,L. Wrenn, Conclusive evidence for internal dielectric charging anomalies on geosynchronous communications spacecraft, J. Spacecr. Rockets 32 (1995) 514-520.

[4] N., Iucci, A.E. Levitin, A.V. Belov, E.A. Eroshenko, N.G. Ptitsyna, G. Villoresi, G.V. Chizhenkov, L.I. Dorman, L.I. Gromova, M. Parisi, M.I. Tyasto, V.G. Yanke, Space weather conditions and spacecraft anomalies in different orbits, Space Weather 3 (1) (2005) S0100110.1029/2003SW000056.

[5] R. Hanna, T. Paulmier, P. Molinié, M. Belhaj, B. Dirassen, D. Payan, N. Balcon, Radiation induced conductivity in Teflon FEP irradiated with multienergetic electron beam, IEEE Trans. Plasma Sci. 41 (12) (2013) 3520-3525.

[6] J.F. Fowler, X-ray induced conductivity in insulating materials, Proc. R. Soc. Lond. A Math. Phys. Sci. 236 (1207) (1956) 464-480.

[7] B. Gross, R.M. Faria, G.F. Ferreira, Radiation induced conductivity in Teflon irradiated by X-rays, J. Appl. Phys. 52 (2) (1981) 571-578.

[8] A.P. Tyutnev, V.S. Saenko, I.A. Smirnov, E.D. Pozhidaev, Radiation-induced conductivity in polymers during long-term irradiation, High Energy Chem. 40 (5) (2006) 319-330.

[9] T. Paulmier, B. Dirassen, D. Payan, M. Arnaout, Analysis of charge transport and ionization effect in space-used polymers under high-energy electron irradiation, IEEE Trans. Plasma Sci. 45 (8) (2017) 1933-1937.

[10] T. Paulmier, B. Dirassen, M. Arnaout, D. Payan, N. Balcon, Radiation-induced conductivity of space used polymers under high energy electron irradiation, IEEE
Trans. Plasma Sci. 43 (No. 9) (September 2015) 2907-2914.

[11] G.M. Yang, G.M. Sessler, Radiation-induced conductivity in electron-beam irradiated insulating polymer films, IEEE Trans. Electr. Insul. 27 (4) (1992) 843-848.

[12] R. Hanna, T. Paulmier, P. Molinie, M. Belhaj, B. Dirassen, D. Payan, N. Balcon, "Radiation induced conductivity in space dielectric materials, J. Appl. Phys. 115 (033713) (2014), http://dx.doi.org/10.1063/1.4862741.

[13] T. Paulmier, B. Dirassen, D. Payan, M. Van Eesbeek, "Material charging in space environment: experimental test simulation and induced conductive mechanisms, IEEE Trans. Dielectr. Electr. Insul. 36 (3) (2009) 682-688.

[14] J.I. Vette, The AE-8 trapped electron model environment, nation space science data center (NSSDC) world data center a ror rockets and satellites (WDC-A-R\&S), (January 1991) 91-24.

[15] R. Hanna, T. Paulmier, P. Molinie, M. Belhaj, B. Dirassen, D. Payan, N. Balcon, "Radiation induced conductivity in space dielectric materials", J. Appl. Phys. 115 (033713) (2014), http://dx.doi.org/10.1063/1.4862741.

[16] A. Roggero, T. Paulmier, B. Dirassen, S. Lewandowski, E. Dantras, C. Tonon, S. Dagras, D. Payan, Electrical conductivity of a space-used silicone elastomer: evolution under electron irradiation, J. Spacecr. Rockets 53 (2016) 1114-1118, http://dx.doi.org/10.2514/1.A33452 Special Issue on Materials in a Space Environment.

[17] Aurélien Roggero, Eric Dantras, Thierry Paulmier, Claire Tonon, Simon Lewandowski, et al, Dynamic Glass Transition of Filled Polysiloxane upon Electron irradiation.", Journal of Non-crystalline Solids, vol. 455, Elsevier, 2017, pp. 17-23.

[18] T. Paulmier, R. Hanna, M. Belhaj, B. Dirassen, D. Payan, N. Balcon, C. Tonon, E. Dantras, A. Bernès, Aging effect and induced electric phenomena on dielectric materials irradiated with high energy electrons, IEEE Trans. Plasma Sci. 41 (12) (2013) 3422-3428.

[19] A. Rose, "Recombination processes in insulators and semiconductors, Phys. Rev. 97 (Jan. 1955) 322-333. 